\title{
Automated perimetry in patients with choroidal
} metastases

\author{
Firas M Rahhal, David H Abramson, Camille A Servodidio, Hilary S Abramson
}

\begin{abstract}
Aims/Background-Recent work using computerised perimetry has documented predictable visual field defects in patients with choroidal melanoma. Despite the higher frequency of occurrence of choroidal metastases, relatively little is known about their exact effects on visual performance, specifically with respect to visual field. This study is a pilot retrospective analysis of the results of automated perimetry testing in patients with choroidal metastatic disease.

Methods-The Humphrey field analyser was used to perform visual field tests on 15 eyes in 11 patients diagnosed with choroidal metastases. All 11 primary tumours were carcinomas originating in the breast (seven), lung (one), kidney (one), stomach (one), and prostate (one). All patients had either central 30 and/or peripheral 30-60 threshold strategies.

Results-Each of the 15 eyes tested had a demonstrable visual field defect. Eight eyes $(53 \%)$ had absolute scotomas and seven $(43 \%)$ had relative scotomas; the defects did not consistently correspond in size or location to the tumour as depicted by ophthalmoscopy or ultrasonography. This is in contradistinction to automated perimetry results in patients with choroidal melanoma. Three of the four eyes (75\%) which were retested after treatment of the metastases showed improvement of the visual field. Seven of nine eyes $(77 \%)$ that initially had reduced visual acuity had improvement after treatment. This clinical improvement corresponded well to tumour shrinkage and resolution of subretinal fluid.
\end{abstract}

Oepartment of York Hospital-Cornell Medical Center, New

York, USA

F M Rahhal

D H Abramson

C A Servodidio

Harvard College, Cambridge, USA H S Abramson

*Current address:

Department of

Ophthalmology,

University of California

San Diego School of

Medicine, La Jolla, CA, USA.

Correspondence to: Firas M Rahhal, MD, 2212 Carol View Drive, No 202 . Cardiff, CA 92007, USA.

Accepted for publication 21 December 1995
Computerised visual field testing has become
the new standard for diagnosing and following

Computerised visual field testing has become
the new standard for diagnosing and following cases.

(Br f Ophthalmol 1996; 80: 309-313) patients with glaucoma. ${ }^{1-3}$ Only recently has attention been given to the visual fields of patients with choroidal melanoma before ${ }^{34}$ and after treatment. ${ }^{5}$ Abramson described predictable visual field defects in 98 choroidal melanoma patients before treatment. These choroidal melanoma patients had an absolute scotoma surrounded by a relative scotoma corresponding to, but larger than, the area of the tumour. ${ }^{4}$ The defects frequently continued down and across the midline. After treatments where the eye was salvaged the scotoma became larger in $50 \%$ of cases and smaller in $50 \% .^{5}$ While the central 30 degree threshold strategy was the best in detecting these changes, they had been previously described in screening strategies. ${ }^{6}$

Although metastatic ocular disease is more common than primary uveal melanomas, there are no publications detailing the results of automated perimetry in patients with choroidal metastatic disease. The lack of information on visual fields in this patient population may be the result of the patients' overall medical condition and their often complicated and exhausting clinical protocols, both of which may preclude the opportunity or need for visual field testing. An alternative reason is that ophthalmologists are rarely (if ever) the primary clinical case managers of these patients.

We have seen patients whose first manifestation of cancer was a visual field defect caused by a non-rhegmatogenous retinal detachment. We wondered if the automated visual fields of patients with choroidal metastases were, in fact, consistently abnormal, and, if so, whether or not they were predictable and followed a pattern. We were also interested in how the visual fields would change after treatment of the metastases. The decision to treat metastatic ocular disease relates to function, since rarely (if ever) will the treatment of ocular metastases affect patient survival. Since visual field is an important measure of overall visual function, it would be valuable to know whether or not field defects improve following treatment in order to adequately counsel patients and their referring physicians. Furthermore, knowledge of the visual fields of patients with metastatic disease will allow comparison with those of patients with choroidal melanoma, and if different patterns of field defects exist they may be useful in difficult cases where ocular diagnosis is uncertain. The current study is, therefore, a pilot retrospective analysis of patients with metastatic choroidal disease who had undergone Humphrey visual field testing. 


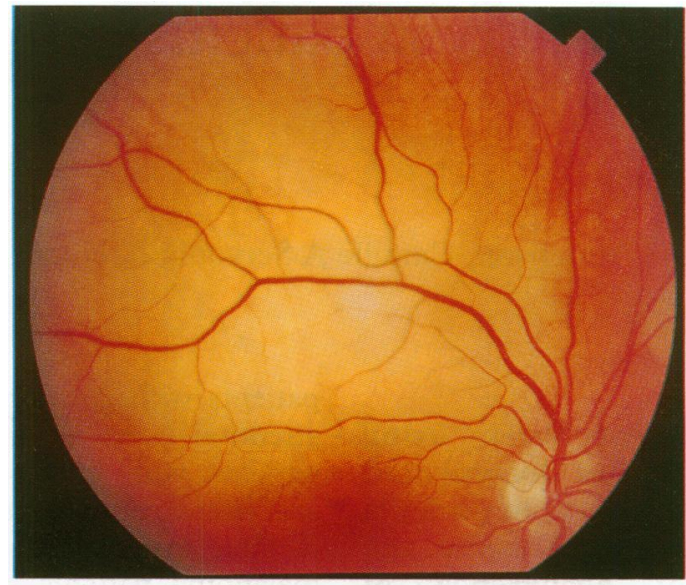

Fig $1 \mathrm{~A}$

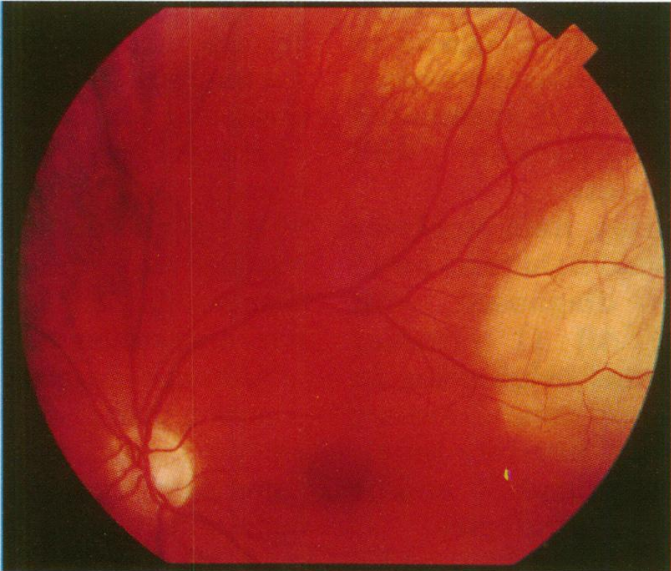

Fig $1 B$

\section{Patients and methods}

Humphrey computerised visual fields were performed on 15 eyes of 11 patients diagnosed with metastatic carcinoma. Seven patients were female and four were male. The average age at presentation was 60 years old (range 42-72). The primary tumour was breast carcinoma in seven patients, and one each of lung, gastric, prostate, and renal cell carcinoma. Seven patients had unilateral metastases (four left eyes and three right eyes) and four of the 11 patients had bilateral disease. Two of the patients with unilateral disease subsequently developed metastases to the other eye but only involved eyes at the time of initial examination were considered in this study.

Perimetry was performed using the Humphrey field analyser (model 630) with the appropriate near vision correction for the central 30 degrees and without correction for the peripheral 30-60 degrees. The central 30 tests employed included either the central 30-1 or 30-2 'quantify defects strategy'. The same strategy was performed for the peripheral 30-60-1 or 30-60-2 tests. An absolute scotoma was defined as three or more contiguous points of zero decibels (or 10000 apostilbs). A relative scotoma was defined as three or more contiguous points with a defect of greater than or equal to $6 \mathrm{~dB}$ (or 2500-9999 apostilbs). Visual field improvement was defined as either decrease in size or depth of the scotoma.

Visual acuity was always measured using best correction (before and after treatment). Improvement was defined as greater than or

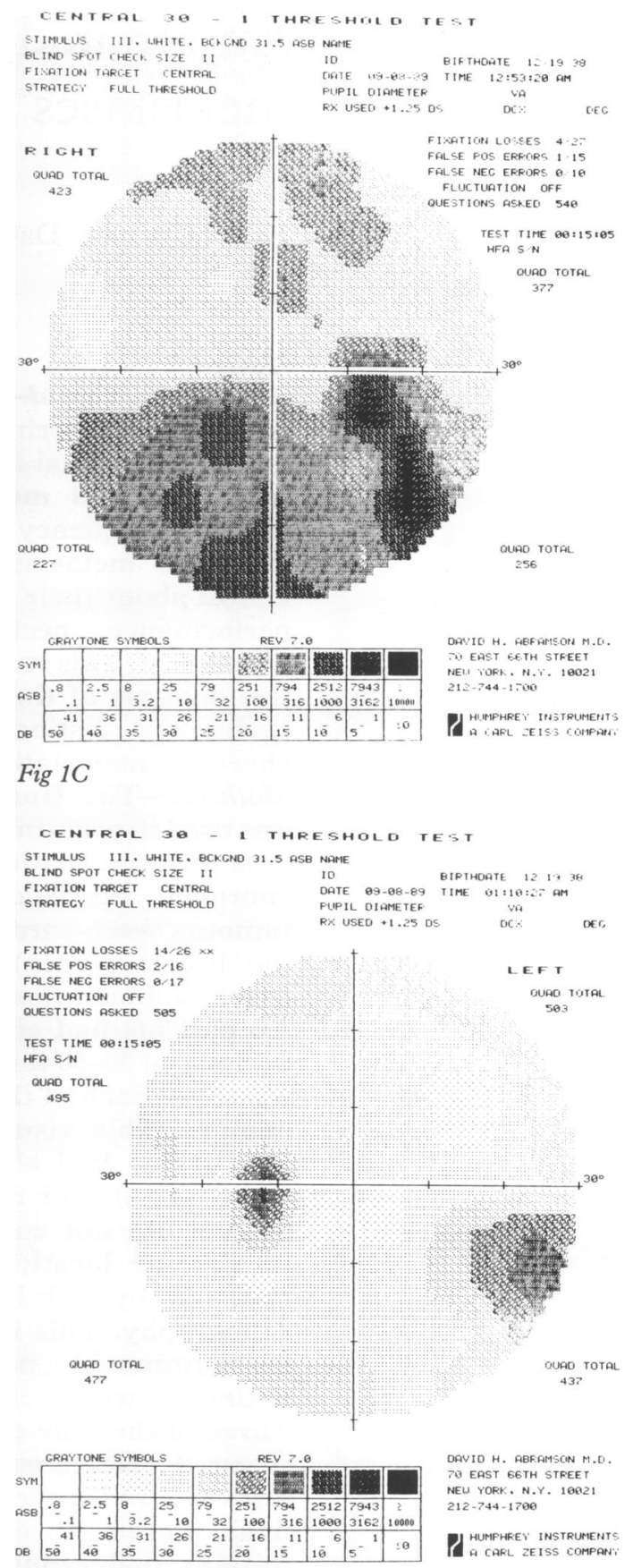

Fig $1 D$

Figure 1 Fundus photographs of right eye $(A)$ and left eye $(B)$ and visual field $(C, D)$ of a patient with bilateral choroidal metastases demonstrating correspondence of tumour size and location with scotomas.

equal to two lines of Snellen acuity improvement.

\section{Results}

Visual fields of 15 eyes of 11 patients with metastatic disease were reviewed retrospectively. The Humphrey visual field test was abnormal in all 15 eyes (100\%). Eight eyes $(53 \%)$ had absolute scotomas and seven $(47 \%)$ had relative scotomas. Ten of the 15 eyes $(67 \%)$ had ophthalmoscopic and ultrasonographic evidence of serous retinal detachment on presentation.

Although the majority of the visual field defects did not correspond to the size and location of the choroidal tumour mass, Figure 1 


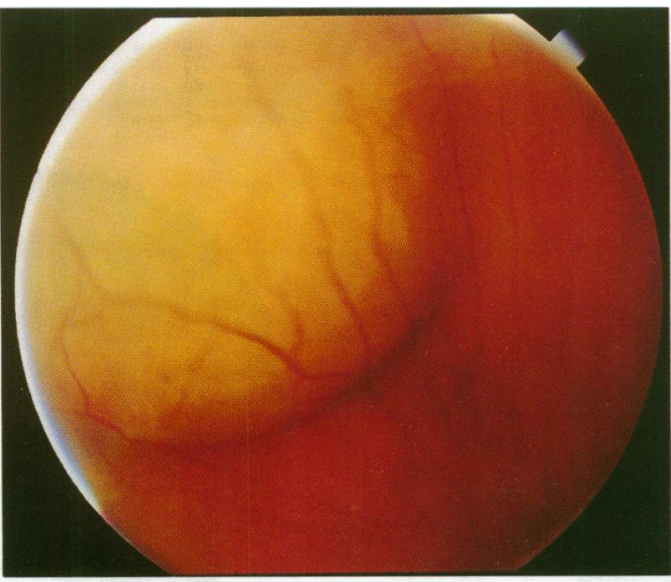

Fig $2 A$

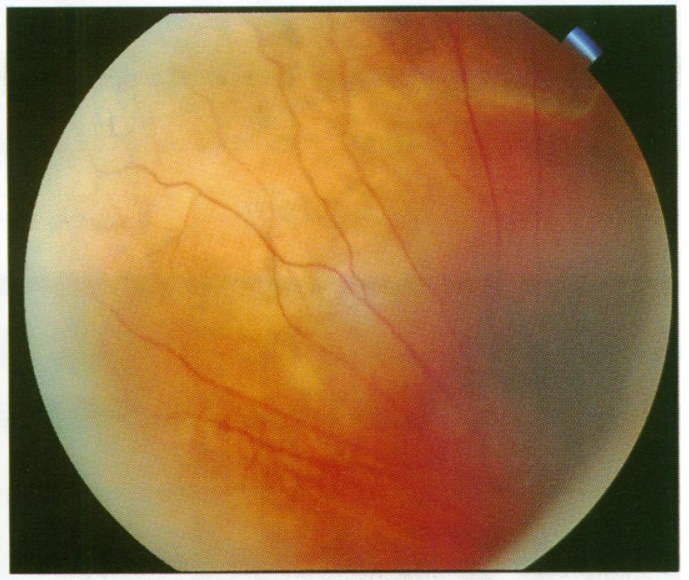

Fig $2 B$

shows one of the exceptions in a patient whose bilateral visual field loss corresponded well with the tumour as viewed ophthalmoscopically.

Four patients were retested following treatment with local irradiation, systemic chemotherapy, hormonal therapy, or a combination of these. Three of the four (75\%) retested eyes (in four patients) demonstrated improvement in the visual fields following treatment. The improvement was either a shrinkage in the size or depth of the scotoma. Figure 2 shows one patient's visual field and fundus photographs before and after treatment. This patient's visual field loss demonstrated some correspondence with the location of the tumour, but there was also some diffuse peripheral loss. Both areas improved with treatment. Visual fields performed before, during, and after treatment of another patient are demonstrated in Figure 3, along with the corresponding B-scans. This patient's field loss did not correspond with the tumour location ophthalmoscopically (fundus photographs not available). The patient's visual field worsened initially (Fig 3B), during therapy, before it ultimately improved (Fig 3C). The significant improvement in false negative errors between the first (eight out of 14) and second (one out of 10) tests, without concomitant improvement in field loss, gives good evidence that the final improvement was not simply an artefactual learning effect of the Humphrey automated perimeter.

Of the nine eyes in six patients that presented with decreased visual acuity, seven

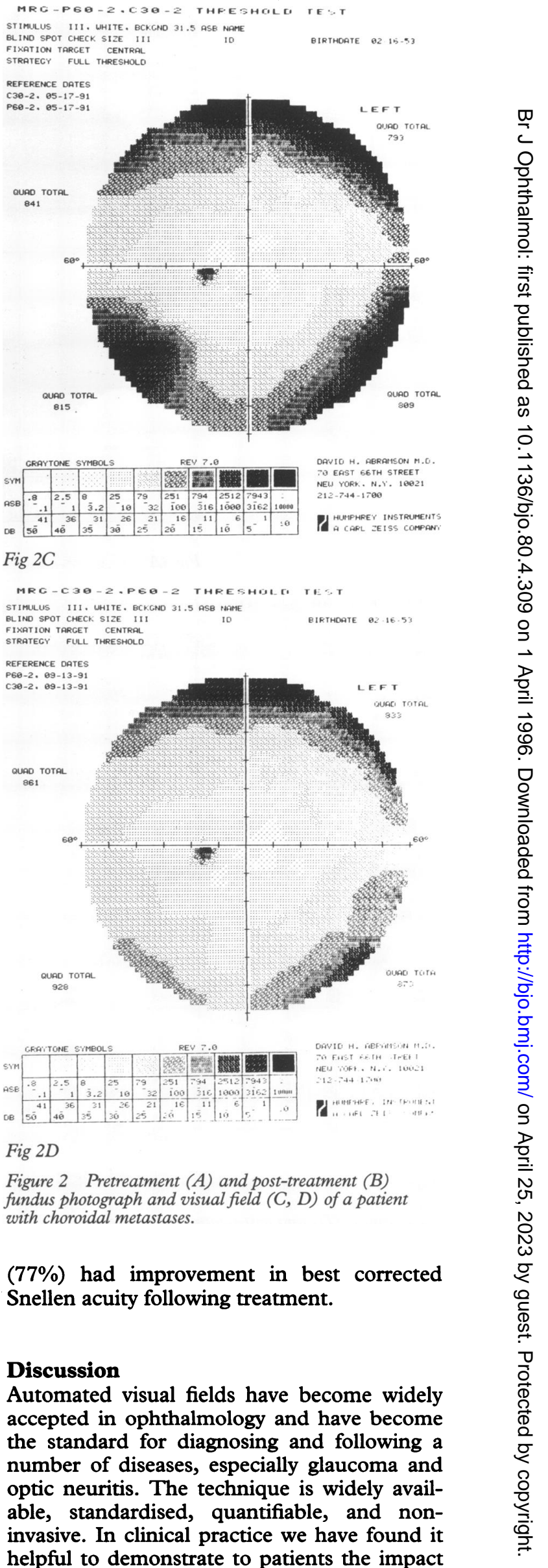
of the ocular melanoma and to aid them and the clinician when deciding between radiation and enucleation.

In choroidal melanoma all patients we have been able to test demonstrated an absolute 

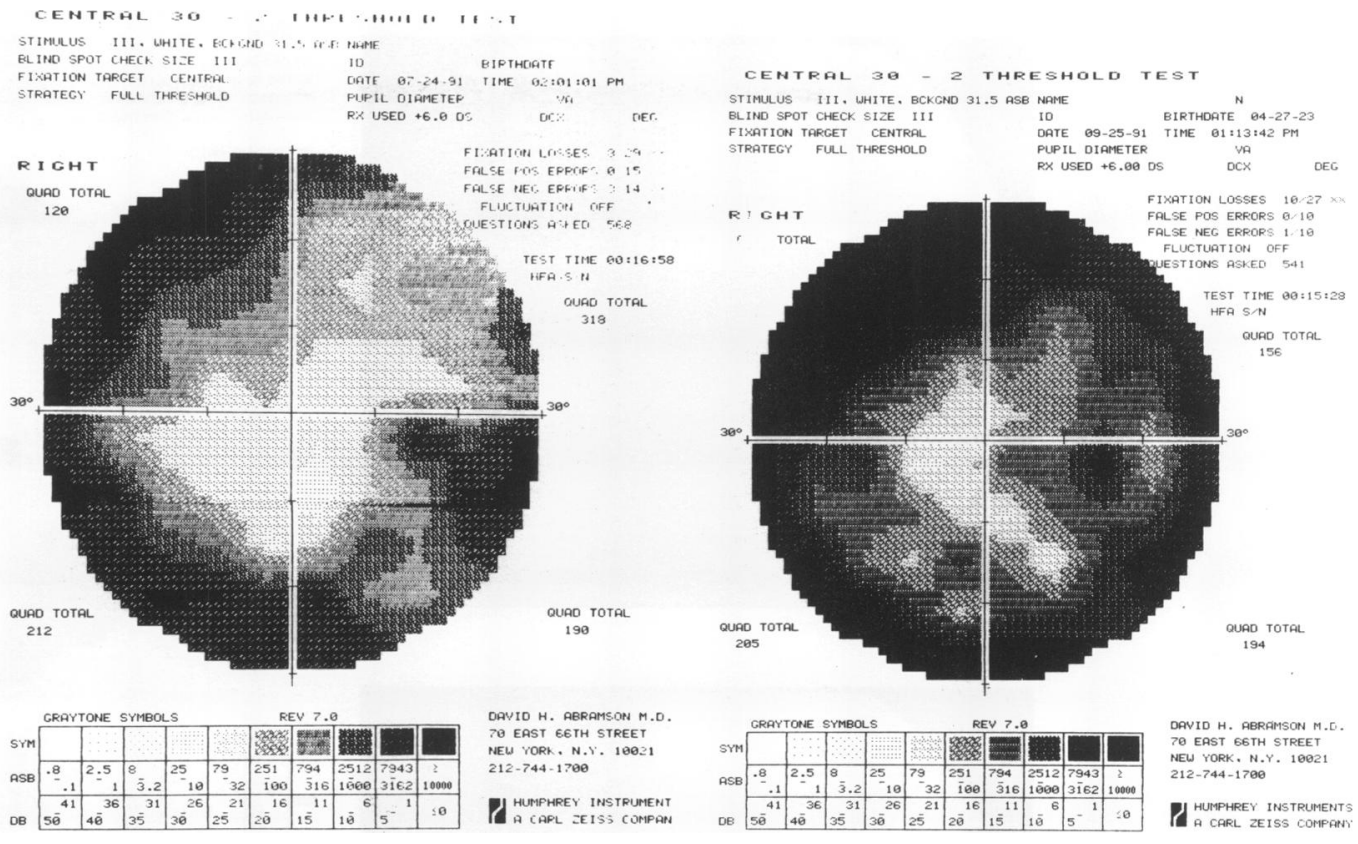

field defect and surrounding relative field defect that corresponded precisely with the anatomical location of the tumour but was usually larger than the tumour itself. This relative scotoma, probably caused by the secondary serous detachment or prior localised detachment, frequently extends above (corresponding to the fluid which pools below) and may go across either or both the horizontal and
Fig $3 B$

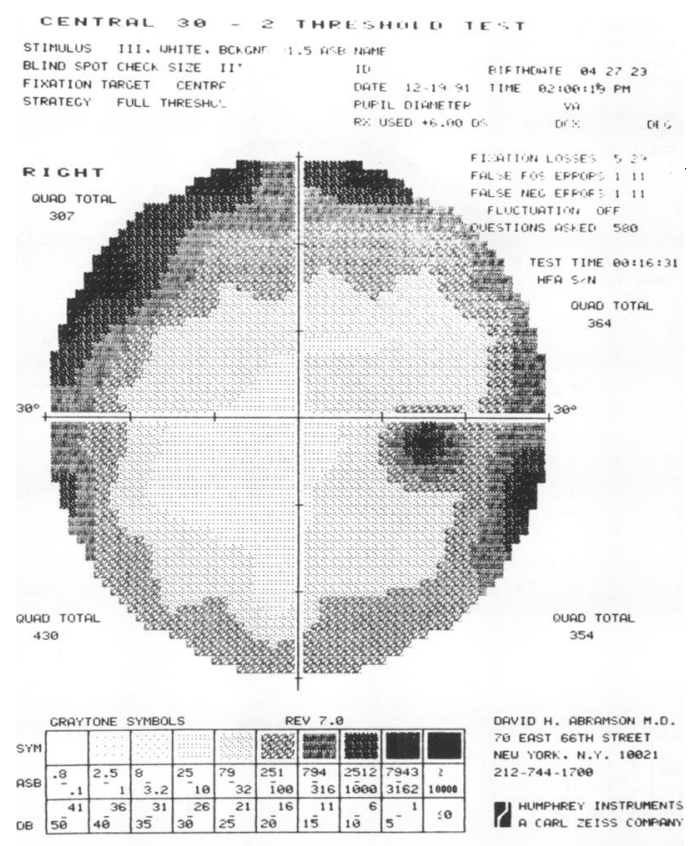

Figure 3 Humphrey visual field results of a patient before $(A)$, during $(B)$, and after treatment $(C)$. Corresponding pretreatment (D) and post-treatment (E) B-scan

sonograms are also shown. Note the tumour shrinkage and improvement in visual field after treatment. Despite the significant improvement in false negative responses between False negative responses remained stable between the second and third tests, but the visual field improved markedly.

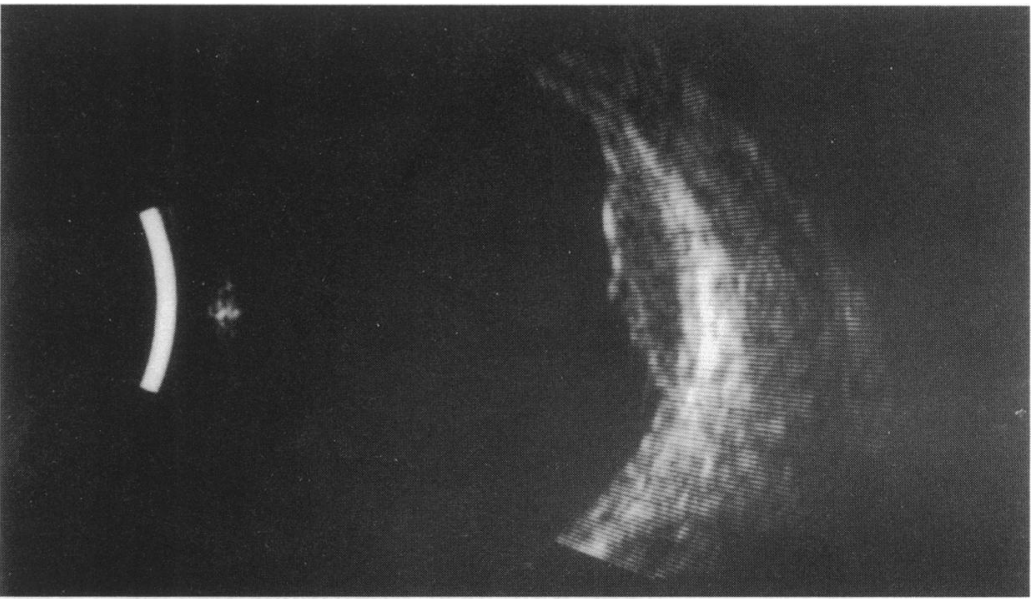

Fig $3 D$

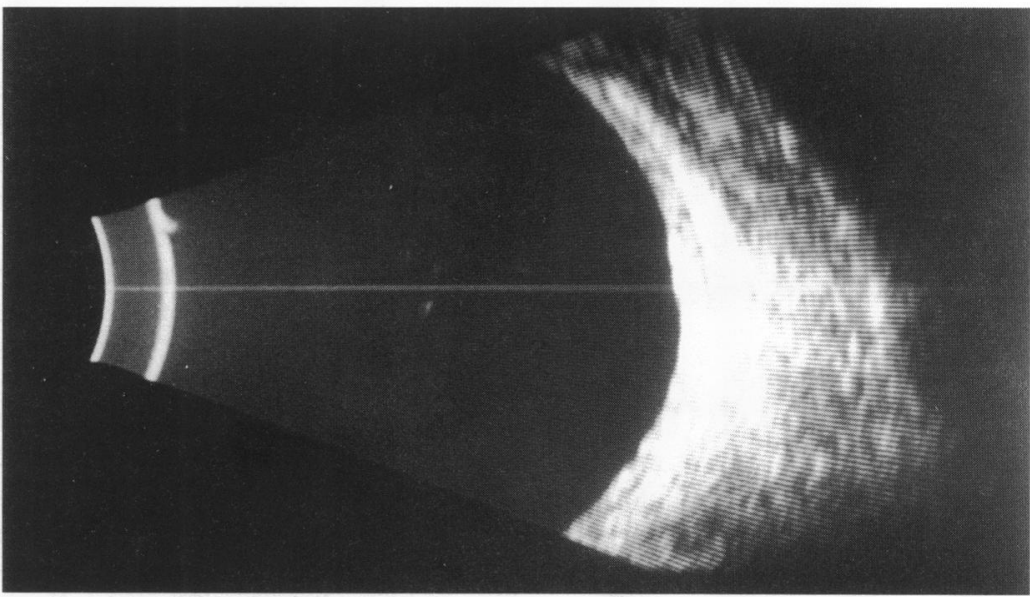

Fig $3 E$

vertical meridians. ${ }^{4}$ After brachytherapy the field increases in $50 \%$ of patients and decreases in $50 \%$, but the absolute scotoma never disappears. ${ }^{5}$ Visual acuity improvement (short term) frequently occurs as the secondary serous detachment disappears.

In this pilot study we were able to perform Humphrey visual fields without difficulty on patients with metastatic disease. Since the 
majority of patients with metastatic choroidal disease had tumours in the posterior pole, we were able to shorten testing by using central 30 ( 1 or 2 ) strategies. All tested patients had abnormal fields but this may represent a selection bias because patients with metastatic ocular disease who are referred to an ophthalmic oncologist are frequently referred because of decreased visual functioning. Three of the four patients $(75 \%)$ retested with automated perimetry following treatment did show improvement in their field defect. Although it could be argued that some improvement may simply be a learning effect of automated perimetry, the sequence of fields shown in Figure 3 strongly suggests the improvement is real. Furthermore, the improved visual function documented with Snellen visual acuity was quite consistent with that observed with perimetry $(77 \%$ of eyes had better acuity following treatment). Although these sample sizes are small, this documentation of visual improvement could justify treatment of choroidal metastases, in selected cases, if the goals of treatment are related to visual function. Our results suggest that visual fields may be useful in deciding which eyes should be treated, and in explaining to the patient and referring physician exactly what improvement can be expected from therapy. A prospective evaluation of patients with metastatic disease would seem appropriate based on this pilot experience.

There were more differences than similarities when comparing visual field defects of patients with choroidal melanoma with those of patients with metastatic choroidal tumours. The similarities were that in all cases of tumours (melanomas or metastatic tumours) visual field defects were demonstrable; the visual field defects appeared larger than the tumour itself and some of these defects became smaller after irradiation. The differences were also significant. In melanomas the visual field defect corresponded exactly with the location of the tumour; in metastatic tumours it usually did not correspond with the location of the tumour. This most probably relates to that fact that diffuse choroidal infiltration, which is not typically ophthalmoscopically apparent, occurs in metastatic disease and may result in more diffuse field loss. Also, the effect of paraneoplastic syndromes (for example, cancer associated retinopathy) on the visual fields of patients with metastatic carcinoma remains unclear. In melanomas there was always an absolute field defect overlying the tumour while seven of the 15 eyes with metastases did not have an absolute field defect. The reasons for these differences deserve careful future analysis. If these differences are shown to be correct in a larger series, then automated perimetry may be helpful in distinguishing amelanotic melanomas from some metastases.

This work was presented in part at the Association for Research in Vision and Ophthalmology Meeting, May 1994.

The authors have no proprietary interest in the topic or materials addressed in this paper.

1 Heijl A, Drance SM. A clinical comparison of three computerized automated perimeters in the detection of glaucoma defects. Arch Ophthalmol 1981; 99: 832-6.

2 Beck RW, Bergstrom BJ, Lichter PR. The clinical comparison of visual field testing with a new automated perimeter, the Humphrey field analyzer, and the Goldmann perimeter. Ophthalmology 1985; 92: 77-82.

3 Trope GE, Britton R. A comparison of Goldmann and Humphrey automated perimetry in patients with glauHumphrey automated perimetry in patien

4 Abramson DH. Computerized visual field defects of choroidal melanomas. Glaucoma 1988; 10: 39-48.

5 Palazzi M, Abramson DH, Myers D, McCormick B. Computerized visual fields in uveal melanomas following treatment. Glaucoma 1989; 11: 114-24.

6 Meecham WJ, Char DH, Chen GT, Juster R, Castro JR, Stone RD, et al. Correlation of visual field, treatment fields, and dose in helium ion irradiation of uveal melanomas. $\mathrm{Am}$ f Ophthalmol 1985; 100: 658-65. 